\title{
Three case studies involving Leptospira interrogans serovar pomona infection in mixed farming units
}

\author{
B Gummow ${ }^{a}$, J G Myburgh ${ }^{b}$, P N Thompson ${ }^{c}$, J J van der Lugt ${ }^{d}$ and B T Spencer ${ }^{a}$
}

\begin{abstract}
Three case studies involving Leptospira interrogans serovar pomona outbreaks within mixed farming systems in South Africa are described. On 2 farms, pigs constituted the main enterprise with cattle and sheep of secondary importance. On each of these 2 farms, abortion due to L. pomona in sows was confirmed by culture, and antibody titres to pomona were detected in cattle, sheep, horses and dogs. On the 3rd farm, a piggery was of secondary importance to cattle farming. Abortion and death in cows occurred on this farm and serology showed titres to various serovars, including pomona. L. pomona was also isolated from bovine urine, an aborted bovine foetus and kidneys from slaughtered pigs. This particular case study was regarded as clinically atypical in that adult Jersey cattle died of acute leptospirosis in a semiarid region of South Africa. In all 3 case studies, the poor management of pig effluent and of the drinking water and its sources played a pivotal role in the transmission of the disease. Inadequate vaccination of animals against Leptospira and poor record-keeping within the secondary farming enterprises were also contributing factors to the spread of leptospirosis.
\end{abstract}

Key words: abortion, bovine, epidemiology, Leptospira interrogans serovar pomona, leptospirosis, mixed farming units, porcine, water.

Gummow B, Myburgh J G, Thompson P N, Van der Lugt J J, Spencer B T Three case studies involving Leptospira interrogans serovar pomona infection in mixed farming units. Journal of the South African Veterinary Association (1999) 70(1): 29-34 (En.). Department of Animal and Community Health, Faculty of Veterinary Science, University of Pretoria, Private Bag X04, Onderstepoort, 0110 South Africa.

\section{INTRODUCTION}

Leptospirosis is an economically important zoonotic disease affecting most mammals throughout the world. The epidemiology of leptospirosis is complex and because of its multi-host nature, persistence of the disease within any population is usually associated with an intricate ecosystem. To implement effective prevention and control measures, a better understanding of the epidemiology of leptospirosis in a region is essential.

Leptospira interrogans serovar pomona is a bacterial pathogen of worldwide importance in pigs. Pigs are considered the

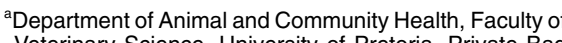
Veterinary Science, University of Pretoria, Private Bag X04, Onderstepoort, 0110 South Africa.

${ }^{b}$ Department of Production Animal Medicine, Faculty of Veterinary Science, Medical University of Southern Africa, PO Box 170, Medunsa, 0204 South Africa.

'Department of Medicine, Faculty of Veterinary Science, University of Pretoria, Private Bag X04, Onderstepoort 0110 South Africa.

dDepartment of Pathology, Faculty of Veterinary Science, University of Pretoria, Private Bag X04, Onderstepoort, 0110 South Africa.

Received: August 1998. Accepted: November 1998 maintenance host $^{5}$. A recent serological survey of slaughter pigs in South Africa revealed a $0.2 \%$ prevalence of antibodies to L. pomona ${ }^{12}$. Antibodies to serovars icterohaemorrhagiae (12.6\%), hardjo $(12.1 \%)$ and bratislava $(7.5 \%)$ were much more prevalent ${ }^{12}$. Outbreaks of acute disease caused by L. pomona are uncommon, but when they occur, may have severe economic consequences for the affected farm $\mathrm{fin}^{1-3,8,10}$. More usually, $L$. pomona causes chronic, asymptomatic disease that may lead to abortion, infertility, and the birth of premature or weak piglets $^{2,4,5,9-11,13}$. Infection by L. pomona in a piggery is usually first recognised when lesions are found in abattoir material ${ }^{8} . L$. pomona has been isolated from pig kidneys and foetuses in South Africa ${ }^{3,8,9}$. The bacterium has also been associated with disease in other species, including cat $\mathrm{tle}^{5,6,9}$, sheep ${ }^{3,15}$, horses ${ }^{16}$ and dogs ${ }^{5} . L$. pomona infections in adult cattle are typically subclinical, but may produce mild clinical signs, whereas the acute haemolytic disease is seen more commonly in calves ${ }^{9}$. Abortions and 'mastitis' are more consistently seen in adult cows'.

Little has been published on the trans- mission and source of leptospirosis in South African mixed farming units. This paper describes 3 epidemiological investigations of the extent and significance of the disease in mixed farming units. The investigations concentrated on the introduction and spread of the disease on the farms to implement effective control measures and prevent further outbreaks.

\section{CASE STUDY 1}

\section{Description of the farm and farm management practices}

The farm was situated on a hill alongside the Olifants River in Mpumalanga Province, South Africa. At the highest point of the hill were the offices, stores, farrowing houses and other buildings necessary to maintain an intensivelyfarmed 300-sow unit (Fig. 1). Roughly half-way down the hill an area of natural pasture began, which was separated from the pig farm by a fence. Within the pasture area was a large crush complex, a freshwater pond and 3 effluent dams (Fig. 1). The pasture was regularly irrigated using the contents of the effluent dams. Cattle, horses and sheep were allowed to graze on this pasture. At the bottom of the pasture was the Olifants River forming the boundary of the farm. A marshy area (vlei) linked the bottom effluent dam to the river (Fig. 1).

The pig farm consisted of 5 farrowing houses and 1 breeding house. Dry sows were kept in open camps without concrete floors adjacent to the farrowing houses. Water for the entire pig farm was pumped from the river to storage tanks at the top of the hill. This water was then gravity-fed into the drinking system and also used to flush the drainage system. The drainage system worked by gravity and consisted of open grid-covered drains that linked the individual pens within the farrowing houses before linking up with similar drains from each house. These drains then ultimately drained via a common open drain, through the pasture, into the effluent dams (Fig. 1).

Sows that aborted in the farrowing pens were immediately moved to the adjacent 


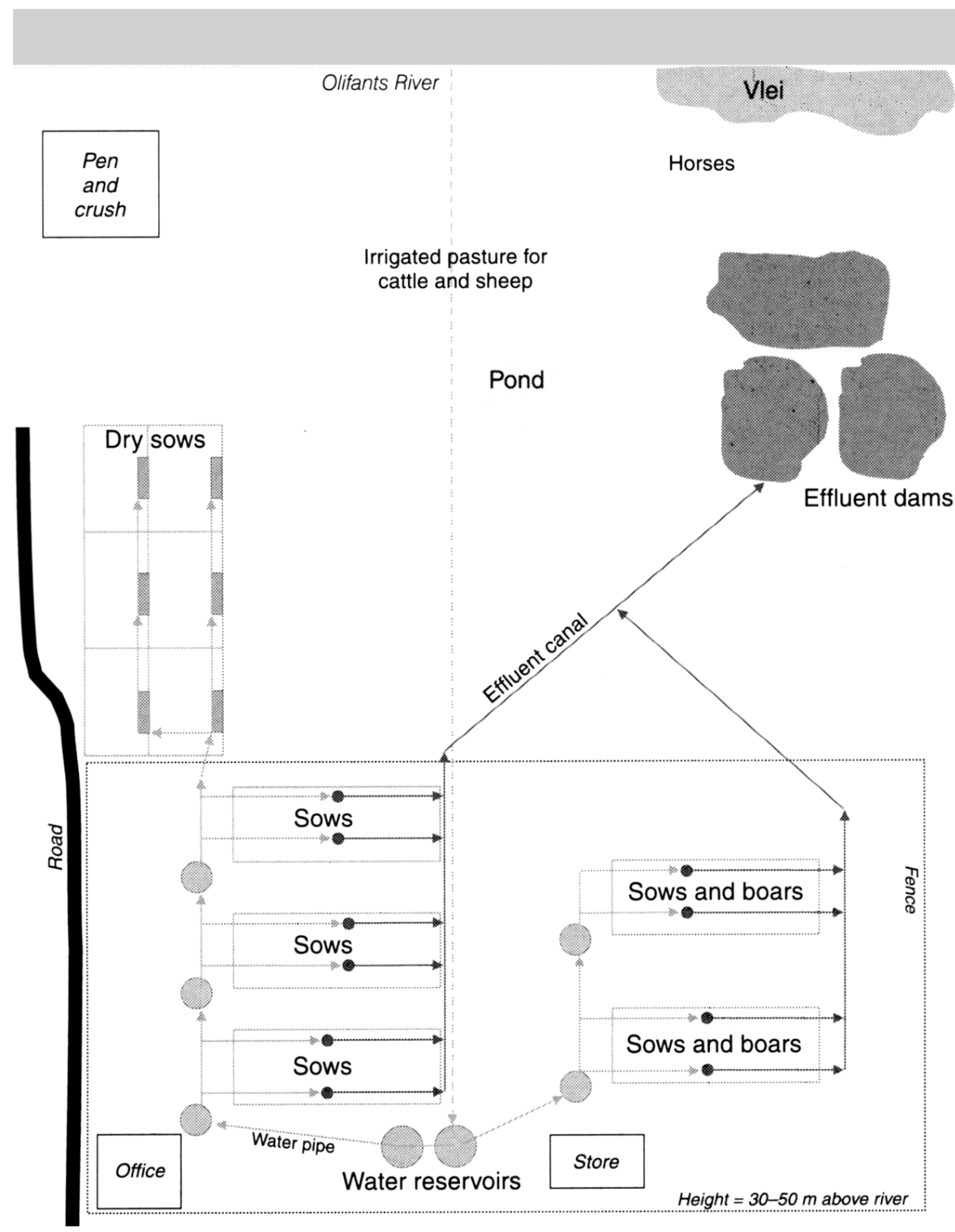

Fig. 1: Map of farm: Case study 1.

open camps to be grouped together with the dry sows. These sows shared common drinking nozzles, the majority of which had pools of water and/or mud around them. Sows to be serviced were moved from the open pens to the breeding house, where the boars were kept and from there via the open pens into the farrowing houses. Newly acquired gilts were not isolated but were placed together with the pregnant sows in the open camps.

\section{Case history and epidemic pattern}

It was determined that 20 gilts had been introduced onto the farm on 12 November from a known L. pomona-infested area in the Eastern Cape. They were placed together with the pregnant sows. In December, 1 sow aborted; in January, 2 sows; in February, 15 sows, and in March 16 sows. On 12 March, treatment with dihydrostreptomycin was carried out. In April, 8 sows aborted; in May, 1 sow, and by June abortions had stopped. A total of 43 abortions were recorded (Fig. 2).

Record-keeping of the other farm animals was so poor that it could not be determined to what extent abortions had occurred within the sheep and cattle populations. However, the authors did note at least 2 cattle abortions while working on the farm. The cause of these abortions was never established.

\section{Diagnostic procedures and results}

A provisional diagnosis of leptospirosis was made based on the abortion storm described above. To confirm this, bacteriological samples were taken from 6 aborted porcine foetuses and 4 slaughter pigs with macroscopic kidney lesions.

L. pomona was isolated from the organs of 5 of the aborted foetuses necropsied and from all 4 slaughter pigs selected on the grounds of macroscopic kidney lesions.

In addition, 20 sows that had recently farrowed or had aborted and 32 randomly selected slaughter pigs were tested serologically for leptospirosis. After confirmation that the causative organism for the abortions was L. pomona, a further 170 cattle, 153 sheep, 13 horses, 4 mice and 2 dogs were tested serologically for antibodies to this serovar ${ }^{3}$. At this point treatment of all pigs on the farm with $30 \mathrm{~m} \ell$ dihydrostreptomycin was carried out.

Of the 170 cattle tested, 88 (52\%) had positive antibody titres (i.e. $>160)$ to $L$. pomona; of the 13 horses tested, 5 (39\%) had positive titres; of the 153 sheep tested, $2(1.3 \%)$ had positive titres and both dogs tested had positive titres. Of the 52 pigs tested, $9(17 \%)$ had positive antibody titres. The cattle were tested for brucellosis at the same time and it was found that, of 22 that had positive titres for brucellosis, 9 were positive concurrently for leptospirosis.

After serological evidence of pomona in the other animal species on the farm was found, a hypothesis about how the disease spread was advanced. To test this hypothesis, mud and water samples were taken from (a) wet areas adjacent to where the dry sows were housed, (b) the open drainage systems, (c) the effluent dams and (d) the drinking water (Fig. 1). These samples were processed in the same way as the organ samples using serial dilutions as described by De Lange et al. ${ }^{3}$. To assess the rodent population on the farm, 40 traps were set in and around the food stores and piggeries. Traps were baited with a mixture of peanut butter and crushed oats and left for a period of 3 days before being examined.

Leptospires (serovar unidentified but not L. pomona) were isolated from the river, open drains and effluent dams. Four mice were caught in the traps, but organisms were not isolated from tissue samples and no antibodies to Leptospira were found.

\section{Discussion}

It has been established that leptospires survive and grow in wet muddy conditions ${ }^{3,6,9,15}$. This investigation showed that these conditions were present within and adjacent to the pig farm. It is also known that the source of infection for animals is water or soil contaminated with infected urine, the infected urine itself or tissues from infected animals $5,10,11,14,15$. The leptospires enter from these sources through cuts or abrasions in the skin or mucosal surfaces, through the conjunctiva, or by inhalation of droplets or 


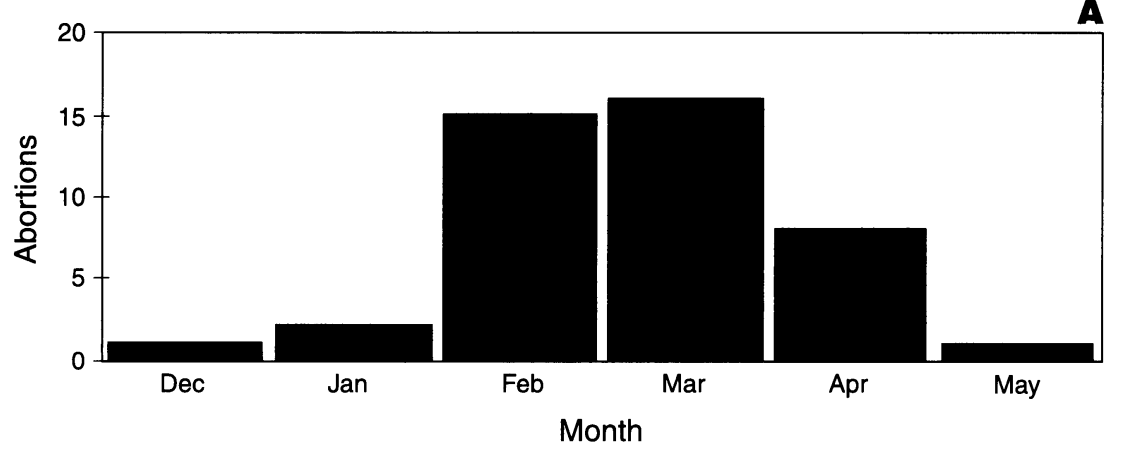

B

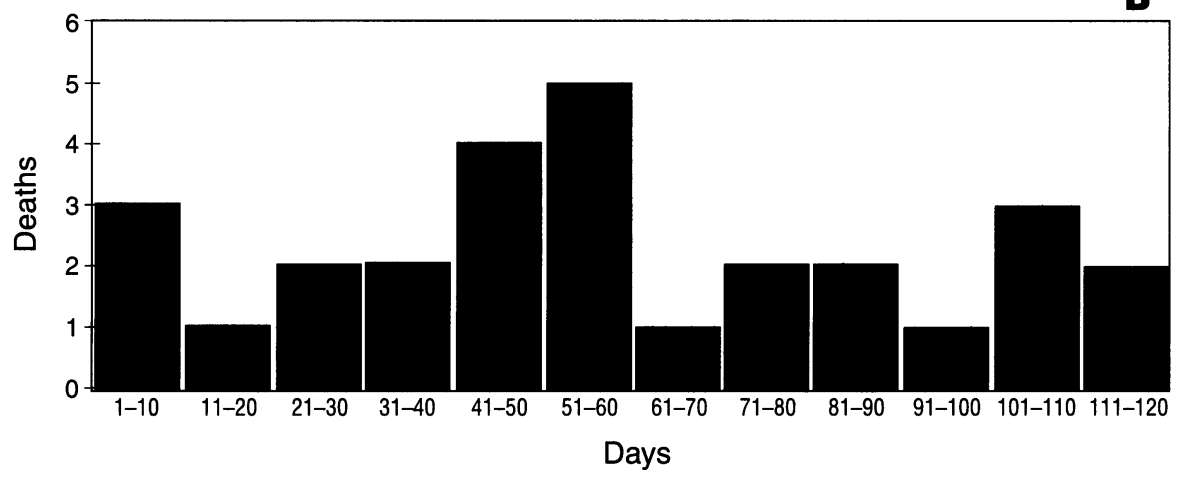

Fig. 2: Epidemic patterns seen for Case study 1: abortions (A); and for Case study 3: deaths $(B)$.

aerosols of fluids containing leptospires ${ }^{5}$. It was therefore postulated that leptospirosis spread throughout the farm in the following manner:

In November, 20 gilts were imported from a known Leptospira-infested area. One or more of these animals was probably infected and shedding organisms in its urine. Upon arrival on the farm, these animals were kept in open camps without concrete floors adjacent to the camps housing pregnant dry sows. Leaking drinking nozzles in these camps were shared by a number of pigs and created an ideal muddy environment in which leptospires could thrive. It is here that the 1st pregnant sow to abort probably became infected with the organisms. This is substantiated by the correspondence of the interval between the introduction of the gilts and that of the 1st abortion, with the incubation period of 14-30 days that is reportedly seen between the onset of an acute infection and abortions in pigs ${ }^{5}$. The 1st affected sow aborted in a farrowing house, thereby effectively contaminating her immediate environment and the open drainage system. This sow was then put back with the dry sows in the open camps, thus completing the 1st cycle of transmission and assisting in infecting other pregnant sows. The abortion storm then escalated as the cycle was perpetuated, to reach a peak in March when veterinary assistance was called in.
During this period, sows had continued to abort in the farrowing houses from where they were moved to the open camps. This probably resulted in contamination of the effluent drainage system and receiving effluent dams. The fact that leptospires were isolated from the effluent drains and dams is evidence that conditions were favourable for their survival and growth in these areas. The leptospires isolated from the dams could not be typed.

The farmer then unwittingly exacerbated the problem by using the contents of the effluent dams to irrigate the surrounding pasture. This may have resulted in contamination of this pasture and any animals grazing on it. Animals grazing in this area gained further exposure to contaminated sources by having free access the dogs) were seen to drink from these dams on several occasions. The fact that animals received considerable exposure to L. pomona is borne out by the high percentage of cattle with antibodies to this serovar.

It is also interesting to note that the cattle, the species with the highest number of serologically positive animals, also most frequently grazed in this area of the farm. Conversely, the sheep, which grazed in this area for only 2 days during the period of investigation, had a substantially lower incidence of infection. Thus, to the effluent dams. Animals (including environmental contamination played a major role in distributing the disease amongst the domestic animals. Owing to the small number of rodents trapped and the fact that those trapped were negative for leptospirosis, it is assumed that wild animals such as rodents probably played no role in distributing the disease.

From the geography of the farm, it is also likely that the farmer contaminated the river flowing on his farm and hence the very water that he was using to wash his farrowing houses. This is supported by the isolation of leptospires from the river. Once the infection had spread from the pigs to the other domestic animals on the farm, these too could play a role in maintaining the infection. The movement of various infected animal species and environmental factors such as the contamination of the river could then contribute to the disease spreading beyond the boundaries of the farm.

Treatment of the pig herd with dihydrostreptomycin had a dramatic effect in curbing the abortion storm, but failure of those involved to appreciate the epidemiology of the disease in this case may have resulted in failure to recognise the cycle of reinfection that had already begun. This could have resulted in possible recontamination of the pig herd at a later stage and in unnecessary stock losses within the other domestic animal populations on the farm.

\section{CASE STUDY 2}

\section{Description of the farm and farm management practices}

This outbreak occurred in a 350-sow unit in the Bronkhorstspruit district, Gauteng Province, South Africa. The piggery was managed as a closed herd. The farmer vaccinated the breeding herd against leptospirosis at 6-month intervals. However, he had adopted the questionable practice of mixing the leptospirosis vaccine with vaccines against parvovirus, erysipelas and Escherichia coli in a single syringe. The farmer also kept cattle and sheep as secondary farming enterprises.

Water from a small river (the Wilge River) was pumped to a cement dam close to the piggery and used in the piggery for both drinking and cleaning purposes. The same water was used as drinking water for the other production animals. Effluent water from the piggery was collected in an effluent collecting dam, and was used to irrigate artificial pastures on which cattle and sheep grazed during the day. Pools of effluent water, to which the dogs had access, also accumulated around the piggery and around the effluent collecting dam. On several occasions, 
the dogs were seen by the farmer to drink this effluent water.

\section{Case history and epidemic pattern}

Three condemned kidneys from slaughtered pigs kept on the farm were submitted for Leptospira isolation and histopathology to the Onderstepoort Veterinary Institute (OVI). The kidneys were condemned at the abattoir owing to the presence of multiple, yellowish-white foci distributed throughout the cortex and medulla (white-spotted kidneys). Samples from the condemned kidneys, examined histologically, showed multifocal, subacute, interstitial nephritis. No bacteria could be demonstrated in sections stained with haematoxylin and eosin, Gram's stain or Warthin-Starry stain.

During the same period, 5 sows $(5 / 350=$ $1.4 \%)$, in late gestation, aborted on the farm and a number of other sows had mummified foetuses. The average litter size in the herd was also smaller than normal for that farm.

\section{Diagnostic procedures and results}

Small pieces of kidney tissue, taken from 3 kidneys condemned at the abattoir, were used to isolate the Leptospira organisms ${ }^{7}$. Leptospira organisms were isolated from all of these samples.

Five aborted pig foetuses and 1 mummified pig foetus were submitted to OVI for Leptospira isolation. Foetal kidney tissue from these was processed as described previously ${ }^{7}$. In addition, a drop of ocular fluid from each foetus was inoculated per tube of media. Leptospira organisms were isolated from the kidneys and from foetal ocular fluid, and the organisms showed positive agglutination reactions with the pomona-positive antiserum.

Thirty-eight serum samples from sows were collected during the outbreak for leptospirosis serology, which was performed using the microscopic agglutination micro-volume technique ${ }^{7}$. Four of the serum samples revealed high antibody titres to L. pomona (median titre $=1 / 2560$ ), and 10 samples $(26.3 \%)$ had low positive titres to $L$. copenhageni (median titre $=$ $1 / 160$ ). One sow had a positive titre to $L$. pomona and $L$ copenhageni.

Based on these results, leptospirosis was diagnosed as the cause of the renal lesions and the abortions. The owner was advised to treat all sows in late pregnancy with a single dose of dihydrostreptomycin $(25 \mathrm{mg} / \mathrm{kg})^{5}$. No further abortions were reported.

Approximately 1 month after the diagnosis had been made, the farm was visited with a view to determining the source

Table 1: Culture results for Leptospira organisms from water samples: Case study 2.

\begin{tabular}{lll}
\hline Source & $\mathbf{p H}^{\mathbf{a}}$ & Positive/negative/contaminated $^{\mathbf{b}}$ \\
\hline & & \\
Surface water from river & 7.47 & Negative \\
Surface water cement dam & 7.58 & Negative \\
Water from outlet of cement dam & 8.28 & Positive: pomona \\
Water on floor in piggery & 7.81 & Contaminated \\
Effluent water from piggery & 7.05 & Contaminated \\
Effluent collecting dam & 8.65 & Contaminated \\
Water trough cattle & 7.16 & Negative \\
Water trough sheep & 8.10 & Negative \\
Dogs drinking place & 6.80 & Contaminated \\
Water from borehole for human use & 7.70 & Negative \\
\hline
\end{tabular}

${ }^{a}$ Where more than one $\mathrm{pH}$ value was available per source, the mean is shown

${ }^{\mathrm{b}}$ Positive = Leptospira organisms isolated; negative = no growth; contaminated $=$ culture overgrown by contaminants.

and extent of the Leptospira outbreak. Blood samples were collected from all the horses $(n=6)$, dogs $(n=6)$ and mature cattle (older than 18 months, $n=19$ ) on the farm, and from 24 randomly selected sheep (roughly $20 \%$ of the flock). Blood samples were also collected from 27 post-weaner pigs. Positive titres to serovar pomona were recorded in 11/19 cattle (58\%), 3/24 sheep (12.5\%) and 3/6 dogs $(50 \%)$. None of the serum samples from the post-weaner pigs and horses reacted positively to any of the serovars.

Water was collected from several sources in and around the piggery (Table 1 ). The $\mathrm{pH}$ of the water samples was determined by means of a Gallenkamp stick pH-meter (Gallenkamp No. 115082). The samples were tested immediately after collection, and the $\mathrm{pH}$ recorded. For culture, a drop of water was added per tube of $5 \mathrm{~m} \ell$ of semisolid EMJH medium, containing $0.5 \mathrm{mg}$ of 5 -fluorouracil per $\mathrm{m} \ell^{7}$. Five replicates were done per water collection point. Leptospira organisms, of the serogroup pomona, were isolated ${ }^{7}$ only from water collected from the outlet of the cement dam. All the other water samples were either overgrown by contaminants or no organisms were demonstrated by dark-field microscopy.

A severe rat problem was present at the time of the outbreak. Kidneys from 11 rats, randomly selected from more than 100 rats caught in and around the piggery, were collected. Specimens were processed as described, but found to be negative.

\section{Discussion}

In this outbreak, the most likely source of infection for the pigs was Leptospirainfected water since the organism was only isolated from water from the outlet of the cement dam.

The water from the nearby Wilge River was pumped directly into the cement dam. It was later determined that a nearby farmer was keeping cattle and pigs directly upstream from the piggery. This farmer was constantly buying and selling animals and recent abortions had occurred in some of his animals. It is possible that infected animals from this upstream property could have contaminated the Wilge River linking the 2 properties. Leptospira organisms were not isolated from the surface water of the Wilge River or from the surface water of the cement dam. In retrospect it is realised that samples should have been collected from the bottom of the stream and from the bottom of the dam, near its outlet, where Leptospira organisms would probably have been concentrated if present ${ }^{5,9,11}$.

Leptospires are delicate organisms, susceptible to desiccation, $\mathrm{pH}$ values outside the range of $6.2-8.0$, salinity, contamination by other bacteria and to some disinfectants and antibiotics ${ }^{11}$. Cultures from water samples are often quickly overgrown by contaminants ${ }^{10}$. A neutral or slightly alkaline $\mathrm{pH}$ favours survival of the organisms ${ }^{5,14}$. Most of the water samples collected on this farm were markedly alkaline (Table 1) and L. pomona was isolated from a water sample with a $\mathrm{pH}$ of 8.28. Water samples collected from the cattle and sheep troughs were negative (Table 1), but the shallowness of the troughs and direct sunlight may have reduced the survival of the Leptospira organisms.

The high median of the serological titres to L. pomona in the sows $(1 / 2560)$ is indicative of recent infections. The significance of the low positive titres to $L$. copenhageni is unknown but may be related to cross-reactions that can occur between $L$. copenhageni and $L$. pomona when using the microscopic agglutination test. None of the young pigs (growers) showed positive titres to Leptospira organisms. These animals were housed in a different building to the pregnant sows but had access to the same water. The 
microscopic agglutination test primarily detects IgM antibodies, while IgG antibodies are responsible for protective immunity ${ }^{5,7}$. Colostral immunity in the growers may have protected them from infection despite the suspect vaccination status of the herd, owing to the farmer mixing 4 different vaccines in 1 syringe.

L. pomona is a recognised bovine and ovine pathogen ${ }^{5,6,9,15}$. As in the previous case, the cattle and sheep on the farm probably became infected by contact with contaminated water from the piggery that was used to irrigate the pastures where they grazed. A low prevalence of positive antibody titres to L. pomona in the sheep $(12.5 \%)$ was recorded as opposed to the cattle $(58 \%)$. De Lange et al. ${ }^{3}$ reported similar findings in a mixed farming unit. These findings support the belief that sheep are more resistant to infection than cattle and seldom suffer from clinical leptospirosis ${ }^{9,15}$. Vermunt et $a l^{15}$ described 3 outbreaks of L. pomona infection in sheep following exposure of the animals to contaminated water that had accumulated in the environment following heavy rainfall. In these outbreaks, neighbouring pig farms were assumed to be the source of organisms.

Clinical signs of disease were not reported in the dogs in this case study either, despite 3 of them having positive antibody titres to pomona (3/6). In dogs, however, canicola is the predominant serovar, and pomona is only occasionally associated with clinical disease ${ }^{5}$. None of the horses showed positive titres to $L$. pomona, which may be explained by the fact that the horses drank water from a borehole but not from the river.

\section{CASE STUDY 3}

This case study describes an atypical outbreak of leptospirosis in a dairy herd. The outbreak involved a variety of leptospiral serovars and was, as far as could be determined, unusual in several respects.

\section{Description of the farm and farm management practices}

The outbreak occurred in a 250 -cow Jersey herd in the northwestern Free State, South Africa. The herd was on a zero-grazing system and fed a total mixed ration. Also situated on the farm was a 60 -sow unit. Unlike the previous 2 case studies, the piggery was of secondary importance to the farmer, with the Jersey herd being his main interest. The calves were housed in groups in close proximity to the piggery. Adjacent to the piggery was a camp where sick cows were kept. Water sources for both the cattle and the pigs included boreholes and seasonal dams.

\section{Case history and epidemic pattern}

The referring veterinarian reported that, over a period of 4 months, 40 cows, representing $16 \%$ of the herd, had died (Fig. 2). Apart from a single 1-year-old animal, only cows over 3 years of age had been affected. No unusual sickness or deaths had been reported amongst the calves. The major clinical signs observed in the affected animals were the acute development of anaemia and icterus. The farmer also noted red urine, presumably haemoglobinuria, in the majority of cases. Twenty percent of the cows that were pregnant at the time aborted. Within 2-10 days after the onset of symptoms, about $75 \%(27 / 40)$ of the sick animals had died, while the rest recovered. A few cows responded temporarily to oxytetracycline at $10 \mathrm{mg} / \mathrm{kg} /$ day for 5 days, and/or diminazene treatment. However, most of these then relapsed and died 3-7 days later. A few sick animals showed small numbers of Babesia bigemina or Anaplasma marginale organisms on blood smears, which may have accounted for the temporary response to treatment in a few cases. However, only 3 of 13 recovered animals bled showed serological evidence of exposure to Babesia using the complement fixation test. Some affected animals had an inflammatory leukogram and there was evidence of liver damage (i.e. raised serum gamma glutamyltransferase concentrations) in individual cases. Normal serum inorganic phosphate levels and liver copper levels helped rule out other haemolytic disorders such as postparturient haemoglobinuria and chronic copper poisoning.

The temporal distribution of mortalities on the farm began with an initial cohort of animals dying, probably due to a common source exposure. This was followed by a propagating epidemic over the next 50 days, after which sporadic deaths occurred (Fig. 2). The propagating epidemic pattern is what one would expect with an infectious disease within a susceptible herd. It can be assumed that after 50 days a sizeable proportion of the herd would have been exposed and those that had not died would have had sufficient immunity to move out of the leptospiraemic phase of the disease and no longer be at risk of dying. This was substantiated by the serological results.

\section{Diagnostic procedures and results}

Leptospiral antibody titres were initially measured in 13 sick or recently recovered cows using the microscopic agglutination test ${ }^{7}$ and it was found that 7 of these animals had a positive titre to 1 or more leptospiral serovars. A subsequent herd test showed that $50 \%(104 / 209)$ of the entire herd had a titre of $1: 100$ or greater to 1 or, more commonly, multiple serovars. Many of these titres were high, with $19 \%(39 / 209)$ of the herd having titres of 1:800 or greater, indicating recent exposure. A rising titre was demonstrated in several animals where a repeat test was done. The predominant leptospiral serovars detected were icterohaemorrhagiae, pomona and bratislava, with hardjo and szwajizak also strongly represented. None of the cows on the farm had ever been vaccinated against leptospirosis.

Leptospiral titres were also measured in several pigs (9/20), where pomona and bratislava were the predominant serovars. None of the farm dogs (0/8) or the rodents captured $(0 / 8)$ on the farm had detectable titres.

Culture of leptospiral organisms ${ }^{7}$ was then attempted from various fluids and tissues of cows, pigs and rodents. Serovar pomona was the only serovar that was isolated, being found in bovine urine (3/12), the kidney of a near-term aborted bovine foetus and several kidneys from slaughtered pigs (6/16).

Based on the serological results, it was decided to vaccinate all cattle 4 months and older with a multivalent, inactivated leptospiral vaccine. Along with this, the farmer was advised about the treatment of any further clinical cases and the human health implications of the disease. Once the entire herd had been vaccinated with a multivalent vaccine containing the serovars icterohaemorrhagiae, pomona, hardjo, grippotyphosa and canicola, no further clinical cases were reported.

\section{Discussion}

This particular outbreak highlighted 3 interesting features. Firstly, it occurred in a relatively dry area of the country, with an average annual rainfall of about $550 \mathrm{~mm}$, at the beginning of the dry season and unlike the previous 2 cases, was not associated with any obvious water source. These facts could mislead a veterinarian into disregarding leptospirosis as a possible diagnosis. In this particular case study it emerged, after the pigs were shown to be positive for Leptospira, that effluent from the piggery had temporarily accumulated in the pen that housed sick cows from the dairy herd, and this was probably the source of exposure for the cattle.

The 2nd unusual feature was that clinical signs were seen only in adult cows, whereas calves are usually far more susceptible to the acute form of the disease. This could further mislead a veterinarian in making a diagnosis. It transpired that, despite their close proximity, the calves had not been ex- 
posed to run-off from either the piggery or the cows. This added circumstantial evidence supports the hypothesis that the effluent was the source of the outbreak.

Thirdly, it is unusual to see such a high incidence of severe clinical disease and mortality in adult cows. In adults the subacute or inapparent forms, as seen in the previous 2 case studies, are far more common and the mortality usually very low ${ }^{9}$. In this outbreak, the severity of the disease was probably due to the sudden exposure of immunocompromised, susceptible cows to high concentrations of organisms in the hospital pen, where animals were suffering from concurrent disease. The concurrent diseases were in most cases anaplasmosis and occasionally babesiosis, which served to mask the clinical signs of acute leptospirosis and make diagnosis difficult.

Although it is likely that serovar pomona was the primary agent involved in this outbreak, active, concurrent infection with serovars icterohaemorrhagiae and bratislava was also shown to occur. It is possible that icterohaemorrhagiae also played a role in the clinical disease and perhaps originated from the rodents. The role played by bratislava is unclear, although it probably originated from the pigs and is unlikely to have been involved in the development of clinical disease.

\section{CONCLUSIONS}

The 3 case studies highlight management factors as the key to L. interrogans pomona transmission and propagation within mixed farming units. The factors included:

1. Failure to test for Leptospira or quarantine new introductions to piggeries

2. Failure to adequately maintain water supply systems and in particular drinking points, resulting in unnecessarily wet environments.

3. Failure to properly manage or maintain open effluent drainage systems by allowing run off of effluent into surrounding areas and free access to effluent by other species.

4. Failure to adequately monitor and keep records for the secondary farming units within the mixed farming system, that might alert the farmer to problems within these units.

5. Improper or no vaccination of animals, and in particular pigs, on these types of farms.

6. Failure to prevent effluent contamination of drinking sources.

The study also supports the findings of Bolt et al. ${ }^{1}$ that rodents do not appear to play an important role in the epidemiology of L. pomona outbreaks.

The role of other serovars in L. pomona outbreaks is difficult to determine and remains inconclusive. Immunocompromised animals are, however, at higher risk, as evidenced in case study 3 , and concurrent infection with multiple serovars and other diseases may make the animals more susceptible to the effects of L. pomona infections.

The purpose of this paper is therefore to make veterinarians aware that in mixed farming operations, especially where suitable environmental conditions exist, the planning of prevention and control strategies must incorporate all the multifactorial aspects of leptospirosis to be effective.

\section{ACKNOWLEDGEMENTS}

We acknowledge the assistance of Ms S M Benjamin, Mr C Bester, Mr J F de Lange, Dr S Herr, Ms C Lötter, Dr M More O'Ferrall, Dr A D Potts, Dr A R Redman, Mr G Schiele, Dr O Swacina, Dr G V Turner, Mr J van der Toorn and Prof. M C Williams.

\section{REFERENCES}

1. Bolt I, Marshall R B 1995 The epidemiology of Leptospira interrogans serovar pomona in grower pig herds. New Zealand Veterinary Journal 43: 10-15

2. Christianson W T 1992 Stillbirths, mummies, abortions and early embryonic death.
Veterinary Clinics of North America: Food Animal Practice 8: 623-639

3. De Lange J F, Gummow B, Turner G V, Redman A R 1987 The isolation of Leptospira interrogans serovar pomona and related serological findings associated with a mixed farming unit in the Transvaal. Onderstepoort Journal of Veterinary Research 54: 119-121

4. Ellis W A, McParland P J, Bryson D G, Thiermann A B, Montgomery J 1986 Isolation of leptospires from the genital tract and kidneys of aborted sows. The Veterinary Record 118: 294-295

5. Faine S 1994 Leptospira and leptospirosis. CRC Press, Boca Raton, Florida

6. Heath S E, Johnson R 1994 Leptospirosis. Journal of the American Veterinary Medical Association 205: 1518-1523

7. Herr S, Hunter P, De Lange J F 1987 Leptospirosis manual: a practical laboratory guide to the serology and isolation of Leptospira. Reproduction Section, Veterinary Research Institute, Onderstepoort

8. Hunter P, Van der Vyver F H, Selmor-Olsen A, Henton M, Herr S, De Lange J F 1987 Leptospirosis as a cause of "white spot" kidneys in South African pig abattoirs. Onderstepoort Journal of Veterinary Research 54: 59-62

9. Hunter P, Herr S 1994 In Coetzer J A W, Tustin R C, Thompson G R (eds) Infectious diseases of livestock in southern Africa. Oxford University Press, Cape Town

10. Kingscote B F 1986 Leptospirosis outbreak in a piggery in southern Alberta. Canadian Veterinary Journal 27: 188-190

11. Mercy A R 1979 Leptospirosis in pigs. Western Australian Department of Agriculture Farmnote No. 6/79

12. Potts A D, Lötter C, Robinson J T R 1995 Serological prevalence of leptospiral antibodies in pigs in South Africa. Onderstepoort Journal of Veterinary Research 62: 281-284

13. Thiermann A B 1987 Swine leptospirosis: new concepts of an old disease. Proceedings of the United States Animal Health Association 91: 491-496

14. Van der Hoeden 1958 Epizootiology of leptospirosis. Advances in Veterinary Sciences 4: 277-339

15. Vermunt J J, West D M, Cooke M M, Alley M R, Collins-Emerson J 1994 Observations on 3 outbreaks of Leptospira interrogans serovar pomona infection in lambs. New Zealand Veterinary Journal 42: 133-136

16. Wood J L N 1994 How important are leptospiral infections as a cause of equine disease? Equine Veterinary Journal 26: 88 